\title{
Continued surprises in the cytochrome $c$ biogenesis story
}

\author{
Elizabeth B. Sawyer ${ }^{1 凶}$, Paul D. Barker ${ }^{\natural}$ \\ ${ }^{1}$ National Laboratory of Biomacromolecules, Institute of Biophysics, Chinese Academy of Sciences, Beijing 100101, China \\ ${ }^{2}$ University Chemical Laboratory, University of Cambridge, Cambridge, CB2 1EW, United Kingdom \\ $\triangle$ Correspondence: beth.sawyer@cantab.net (E.B. Sawyer), pdb30@cam.ac.uk (P.D. Barker) \\ Received March 15, 2012 Accepted March 21, 2012
}

\begin{abstract}
Cytochromes c covalently bind their heme prosthetic groups through thioether bonds between the vinyl groups of the heme and the thiols of a $\mathrm{CXXCH}$ motif within the protein. In Gram-negative bacteria, this process is catalyzed by the $\mathrm{Ccm}$ (cytochrome $c$ maturation) proteins, also called System $\mathrm{I}$. The $\mathrm{Ccm}$ proteins are found in the bacterial inner membrane, but some (CcmE, CcmG, $\mathrm{CcmH}$, and $\mathrm{Ccml}$ ) also have soluble functional domains on the periplasmic face of the membrane. Elucidation of the mechanisms involved in the transport and relay of heme and the apocytochrome from the bacterial cytosol into the periplasm, and their subsequent reaction, has proved challenging due to the fact that most of the proteins involved are membrane-associated, but recent progress in understanding some key components has thrown up some surprises. In this Review, we discuss advances in our understanding of this process arising from a substrate's point of view and from recent structural information about individual components.
\end{abstract}

KEYWORDS cytochrome $c$, heme, Ccm, post-translational modification, cytochrome $b_{562}$, heme binding

\section{INTRODUCTION}

While the detailed mechanisms of many heme enzymes have reached textbook status, the transport and attachment of the cofactor to its end-user proteins remain poorly understood, although there is considerable interest in elucidating these mechanisms (Allen et al., 2003; Kranz et al., 2009; Stevens et al., 2011). A ubiquitous cofactor in biology, heme is involved in many fundamental biochemical processes that involve highly reactive chemical species. When these reactive species are harbored within complex membrane protein assemblies, these processes become hard to trap and characterize. One much-studied and still unresolved question in this area is the chemistry behind heme attachment to polypeptide in cytochromes $c$, one of the staple paradigms for the protein chemist. The fully membrane-localized System I catalyzes the covalent attachment of heme to c-type cytochromes. Despite difficulties in obtaining structural information, significant advances in our understanding of this process have been made through the study of individual components of System I and the use of novel substrate probes to study the system as a whole. The surprising picture that emerges is almost expected: many aspects of heme chemistry appear to be involved in determining heme's own fate.

Cytochromes $c$ are key components of biological electron transport chains of all organisms, and mitochondrial cytochrome $c$ also plays a role in the initiation of apoptosis (Liu et al., 1996). They are characterized by covalent binding of heme via thioether bonds between the heme vinyl groups and the cysteine residues in a $\mathrm{CXXCH}$ motif, where $\mathrm{X}$ represents any amino acid except cysteine (Barker and Ferguson, 1999). The histidine residue in this motif provides an axial ligand to the heme iron and the mature motif is generally helical in the resulting structures, but not $\alpha$-helical. A small number of naturally-occurring $c$-type cytochromes have a modified $\mathrm{CXXCH}$ motif with three or four ' $\mathrm{X}$ ' residues between the cysteines. Biochemically, the stereospecific attachment of heme to c-type cytochromes is a complex process and at least four distinct systems have evolved to catalyze this reaction, and yet a single cysteine thiol can react with a heme vinyl group uncatalyzed in some circumstances. This article focuses on System I, which is found in $\alpha-, \gamma$ - and some $\beta$-proteobacteria, some extremophiles, and plant and red 
algal mitochondria.

\section{CYTOCHROME C MATURATION}

The covalent attachment of heme to c-type cytochromes in the bacterial periplasm involves at least 12 proteins, including the $\mathrm{ccm}$ (cytochrome $\underline{c}$ maturation), $d s b$ (disulfide bond) and trx gene products (Thöny-Meyer et al., 1995; Kranz et al., 1998) and has become the System I paradigm. Both the apo-cytochrome and the heme prosthetic group must be translocated across the cytoplasmic membrane into the periplasm, where heme is attached stereospecifically to the protein. There is significant evidence that reducing equivalents are required to catalyze the process, ostensibly for two purposes. First, it is thought that the $\mathrm{CXXCH}$ motif in the apo-protein is first oxidized to an internal disulfide as it is translocated to the periplasm but then reduced again before reaction with the heme vinyl groups (Barker et al., 1995; Allen et al., 2003); but what is it reduced to? Second, reducing equivalents are also needed to maintain the iron in reduced state during the actual attachment process. On the face of it, this would appear to be required to avoid oxidation of the cysteine thiols to thiyl radicals that can result in deleterious side reactions (Barker et al., 1993; Allen et al., 2003; Fee et al., 2004), but if the cysteine residues were not already reduced would the oxidation state of the iron matter?

The Ccm system has been extensively reviewed, yet its mode of action remains mysterious (Thöny-Meyer, 2002; Kranz et al., 2009; Stevens et al., 2011). In most Gram-negative bacteria, there are nine $\mathrm{Ccm}$ proteins, designated CcmA-I, and DsbD (or CcdA), found encoded in a single locus within the $E$. coli chromosome (Thöny-Meyer et al., 1995) (Fig. 1). The Ccm proteins are integral membrane proteins with functional domains on the periplasmic face of the inner membrane. CcmABCD form a transmembrane ATP-binding cassette (ABC) transporter, in which $C \mathrm{cmA}$ is the ATP-binding subunit, whilst $\mathrm{CcmB}$ and $\mathrm{CcmC}$ are required for localization to the inner membrane (Feissner et al., 2006). The CcmABC complex transports heme across the inner membrane into the periplasm, where $\mathrm{CcmD}$ facilitates its delivery from CcmC to CcmE (Richard-Fogal et al., 2008); the heme is exported with iron in the reduced $\left(\mathrm{Fe}^{2+}\right)$ state.

CcmE is a membrane-associated protein consisting of an $\mathrm{N}$-terminal membrane-anchoring domain, a soluble domain comprising a six-stranded antiparallel $\beta$-sheet (Arnesano et al., 2002; Enggist et al., 2002), and a flexible C-terminal tail. CcmE has been the subject of a great deal of study ever since the observation that heme is transiently covalently attached to it prior to attachment to the recipient cytochrome $c$ (Schulz et al., 1998). It is not clear that the unusual nature of the proposed heme-histidine bond in this transient species (Daltrop et al., 2002b) is the actual reactive species or whether it is a stable adduct formed by quenching a more reactive species during its extraction for analysis. However, based upon this structure it has been proposed that attachment to CcmE is facilitated when the iron is oxidized, and released to the recipient cytochrome $c$ when the iron is reduced (Richard-Fogal et al., 2009). CcmF, CcmH, and Ccml interact to form a stable membrane-integral complex. CcmF delivers reductant to heme on $\mathrm{CcmE}$, whilst CcmG and $\mathrm{CcmH}$, together with $\mathrm{DsbD}$, reduce the cysteines in the $\mathrm{CXXCH}$ motif of the apo-cytochrome (Kranz et al., 2009; Stevens et al., 2011 and references therein). Intriguingly, it has recently been found that $\mathrm{CcmF}$ contains a binding site for a heme molecule that is not incorporated into the cytochrome $c$ but is thought to be required for electron delivery to the reaction complex of $\mathrm{CcmH}, \mathrm{CcmE}$ and the apo-cytochrome (San Francisco et al., 2011). Hence it appears that there are two possible routes for electron delivery to and from the active site: through the $\mathrm{CcmF}$ heme and through the $\mathrm{CcmH}$ dithiol. A full electron audit of the whole process has not been possible so far and a mechanism remains elusive despite a variety of suggestions.

The unfolded recipient apo-cytochrome is translocated across the inner membrane into the periplasm through the $\mathrm{Sec}$ system, which recognizes a leader sequence of 22 amino acids, which is then cleaved from the polypeptide. Once in position, the apo-protein interacts with the various $\mathrm{Ccm}$ proteins and is oriented to facilitate the formation of thioether bonds between the heme vinyl groups and the $\mathrm{CXXCH}$ cysteines. Recent evidence hints that the oxidation state of the cysteines in the motif is neither the expected dithiol nor the intramolecular disulfide, but could involve the formation of a mixed disulfide between the cysteines in the $\mathrm{CXXCH}$ motif and other proteins or small molecules.

\section{WHOLE-SYSTEM STUDIES}

Evidence for the presence of an apo-cytochrome mixed disulfide comes from studies of how the $\mathrm{Ccm}$ system handles variants of its cytochrome substrates in vivo. A modified cytochrome $b_{562}$, which has been engineered to contain the CXXCH motif and forms c-type covalent bonds to heme in the E. coli periplasm (Barker et al., 1995), has proved to be an excellent probe of the substrate specificity of System I (Allen et al., 2009). Unlike almost all other cytochromes $c$, cytochrome ' $c b_{562}$ ' apo- and holo-proteins are stable in vivo and purification is relatively straightforward, enabling characterization. Correctly and incorrectly matured c-type cytochrome products can be distinguished by absorption spectroscopy and characterized by high resolution methods of NMR and mass spectrometry (MS) (Allen et al., 2003; 2009; Sawyer et al., 2010).

Using this cytochrome $c b_{562}$ probe it has been found that the $\mathrm{Ccm}$ apparatus is able to recognize and correctly mature variant cytochromes with cysteine residues within or adjacent to the $\mathrm{CXXCH}$ motif (Allen et al., 2009). This interesting result 


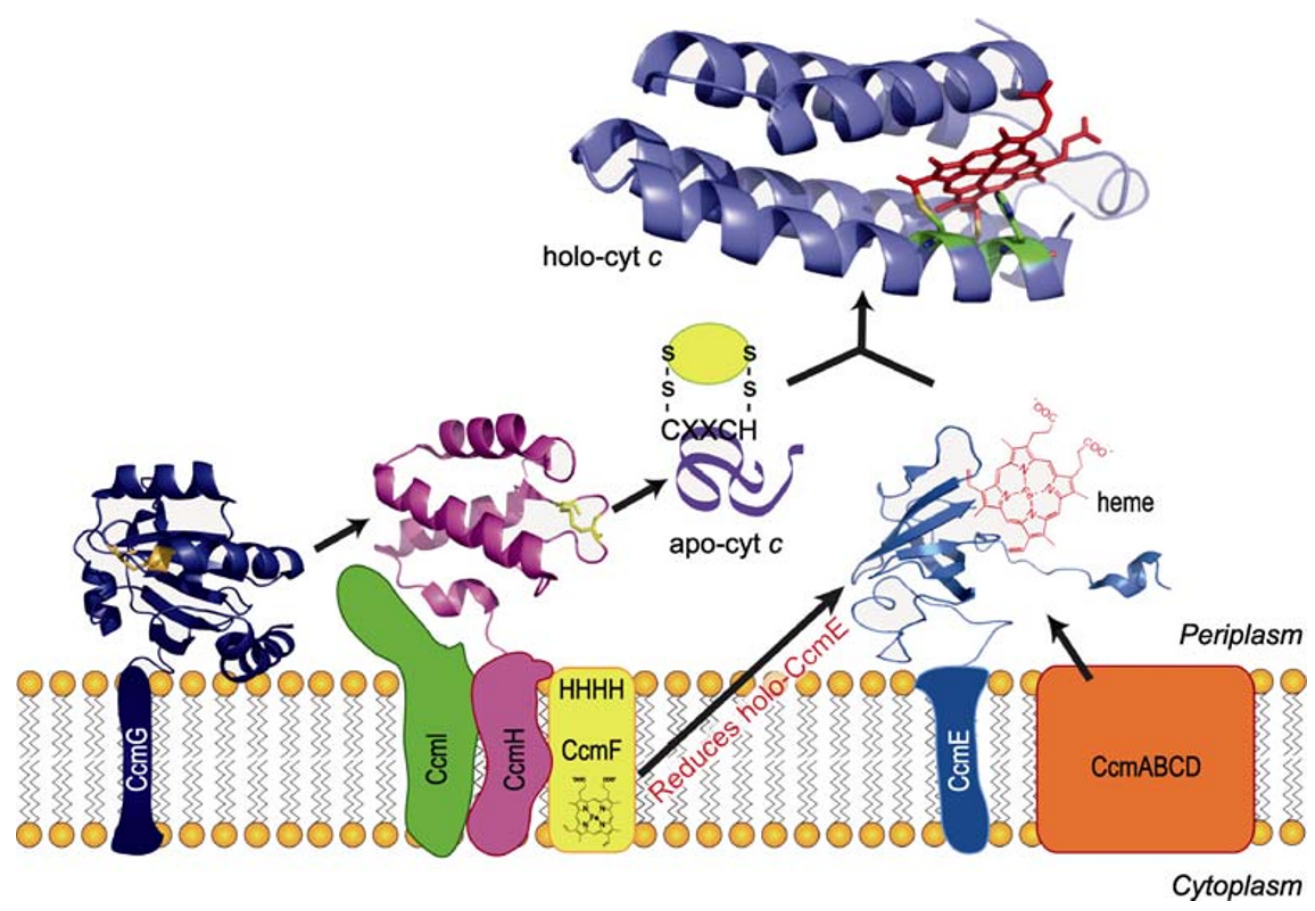

Figure 1. The E. coli cytochrome $c$ maturation $(\mathbf{C c m})$ proteins. The unfolded apo-cytochrome is translocated across the inner membrane into the periplasm via the Sec system. Heme is transported into the periplasm by $\mathrm{CcmABCD}$ and delivered to $\mathrm{CcmE}$, which covalently binds the 2- or 4-vinyl group via an exposed histidine residue. Holo-CcmE is then reduced by $\mathrm{CcmF}$, which contains a $b$ heme that is not incorporated into the apo-cytochrome. The apo-cytochrome probably undergoes a series of thiol redox reactions facilitated by $\mathrm{CcmG}$ and $\mathrm{CcmH}$ before being presented to the heme as a mixed disulfide-likely with a small molecule thiol. Thioether bond formation between the cysteine residues in the protein's $\mathrm{CXXCH}$ motif and the heme vinyl groups then takes place. The structures of the soluble domains of $\mathrm{CcmE}$ (PDB 1SR3), $\mathrm{CcmG}$ (PDB 2B1K), and CcmH (PDB 2HL7) are shown, along with cytochrome $\mathrm{Cb}_{562}$ (adapted from PDB 1QQ3). Catalytically important cysteine residues of CcmG and CcmH are shown in orange/yellow. The cysteines and histidine in the cytochrome $\mathrm{CXXCH}$ motif are shown as sticks and colored by element.

raises the question: why are cysteine residues not observed within the $\mathrm{CXXCH}$ motifs of naturally occurring c-type cytochromes? In fact, cysteine residues (except those within $\mathrm{CXXCH}$ motifs) are rare in the soluble domains of $c$-type cytochromes in general; it seems that despite the fact that cells are able to correctly mature these cytochromes, other evolutionary pressures have led to selection against cysteine. It is likely that this is at least partly due to the need to avoid potential heterogeneity in heme attachment. A further reason may be to ensure that there are no free cysteine thiols available to react with heme iron leading to oxidative damage, or other proteins leading to unwanted dimerization.

Cytochrome $c b_{562}$ variants with unnatural numbers of ' $X$ ' residues $(1,5$ or 6$)$ between the cysteines were also engineered (designated $\mathrm{CX}_{1} \mathrm{CH}, \mathrm{CX}_{5} \mathrm{CH}$, and $\mathrm{CX}_{6} \mathrm{CH}$ ). MS analysis revealed the presence of several species of holocytochrome, differing in mass by multiples of $32 \mathrm{Da}$ (Allen et al., 2009). Interestingly, these "+32" modifications were first observed in 1995 when cytochrome $b_{562}$ was originally converted to a $c$-type cytochrome (Barker et al., 1995). However, since the origin and identity of this extra mass was unknown, and the $\mathrm{Ccm}$ system had yet to be identified as the processor of cytochromes $c$, these species were dismissed as being physiologically irrelevant. It has now become apparent that understanding the origin of these extra mass units may provide insight into the mechanism of cytochrome $c$ maturation by the $\mathrm{Ccm}$ system.

Using high-accuracy MS we were able to show that the extra mass observed is due to sulfur (mass $31.97207 \mathrm{Da}$ ) and not two oxygens (mass $31.9898 \mathrm{Da}$ ) and that it is located on the first cysteine in the $\mathrm{CX}{ }_{n} \mathrm{CH}$ motif, Cys-98 (Sawyer et al., 2010). The "+32" peptides were accompanied in the mass spectra by singly-charged heme fragment ions with one additional hydrogen atom, which seems to be a signature for these sulfur-modified proteins. Furthermore, whenever there was a cysteine residue at the $\mathrm{N}$-terminus of the MS-fragmented peptide, the $y$-ion detected was $51 \mathrm{Da}$ smaller than expected. This is likely to be due to a cysteine persulfide-specific internal fragmentation leading to formation of a propenamide. Interestingly, propenamide $y$-ions were only observed in spectra of sulfur-modified proteins and never in control experiments on normally attached (i.e. no extra mass) heme. These two observations clearly indicate that the persulfide linkage has different chemistry from the normal thio- 
ether heme-protein bond.

What does this tell us about the mechanism of the $\mathrm{Ccm}$ apparatus? The presence of a persulfide bond between the heme and Cys-98 strongly suggests that the apo-protein was modified before being presented to heme in the $\mathrm{Ccm}$ catalytic site. It is likely that the cytochrome $c b_{562}$ modification involves formation of a mixed disulfide, probably with a small molecule thiol. The conformational constraints imposed by having too few or too many ' $X$ ' residues between the two cysteines result in misalignment of the apo-cytochrome substrate and attachment of heme to the wrong sulfur atom in the disulfide. Thus these results suggest that the substrate of the $\mathrm{Ccm}$ apparatus may not be the reduced $\mathrm{CXXCH}$ motif as previously assumed, but rather two disulfides. This has consequences for a redox audit of the mechanism and it is therefore highly pertinent that new redox roles for the $\mathrm{Ccm}$ proteins have recently been described (Richard-Fogal et al., 2009). However, the question remains: why go to all this trouble, since thiols can add to the heme vinyl groups in an uncatalyzed reaction (Barker et al., 1993; Daltrop et al., 2002a; Allen et al., 2003)? In the context of cytochrome $b_{562}$, we have never been able to observe the formation of both covalent linkages in vitro. One idea is that such a unique mechanism has evolved to ensure that both thioether bonds are formed simultaneously in a concerted reaction with an activated heme and that the heme-histidine attachment to $\mathrm{CcmE}$ plays a role in directing the location of a radical during the process. The process certainly has many hallmarks of a highly orchestrated radical transfer reaction.

\section{CONCLUDING REMARKS}

The example of using novel cytochrome $c$ substrates to probe the mechanism of the integral membrane $\mathrm{Ccm}$ proteins shows how membrane proteins may be studied in the absence of structural information or protein complex isolates. The ultimate goal is still to obtain detailed structures of the proteins at each stage of the catalytic cycle. However, in the meantime, while our understanding of the chemistry involved in attaching heme to $c$-type cytochromes continues to grow, we should not be surprised to discover that heme controls its own destiny in this process by harnessing reactive chemistry.

\section{ACKNOWLEDGEMENTS}

This work was supported in PDB's lab by the BBSRC and an EPSRC studentship to EBS. EBS is currently supported by a Chinese Academy of Sciences Fellowship for Young International Scientists (No. 2010Y2SB01) and a grant from the National Natural Science Foundation of China (Grant No. 31150110150). Thanks to Prof. Sarah Perrett for critical reading of the manuscript.

\section{ABBREVIATIONS}

Ccm, cytochrome $c$ maturation; MS, mass spectrometry; NMR, nu- clear magnetic resonance spectroscopy

\section{REFERENCES}

Allen, J.W., Barker, P.D., and Ferguson, S.J. (2003). A cytochrome $b_{562}$ variant with a $c$-type cytochrome $\mathrm{CXXCH}$ heme-binding motif as a probe of the Escherichia coli cytochrome $c$ maturation system. J Biol Chem 278, 52075-52083.

Allen, J.W., Sawyer, E.B., Ginger, M.L., Barker, P.D., and Ferguson, S.J. (2009). Variant $c$-type cytochromes as probes of the substrate specificity of the $E$. coli cytochrome $c$ maturation $(\mathrm{Ccm})$ apparatus. Biochem J 419, 177-184, 2, 184.

Arnesano, F., Banci, L., Barker, P.D., Bertini, I., Rosato, A., Su, X.C., and Viezzoli, M.S. (2002). Solution structure and characterization of the heme chaperone CcmE. Biochemistry 41, 13587-13594.

Barker, P.D., and Ferguson, S.J. (1999). Still a puzzle: why is haem covalently attached in c-type cytochromes? Structure 7, R281-R290.

Barker, P.D., Ferrer, J.C., Mylrajan, M., Loehr, T.M., Feng, R., Konishi, Y., Funk, W.D., MacGillivray, R.T., and Mauk, A.G. (1993). Transmutation of a heme protein. Proc Natl Acad Sci U S A 90, 6542-6546.

Barker, P.D., Nerou, E.P., Freund, S.M., and Fearnley, I.M. (1995). Conversion of cytochrome $b_{562}$ to c-type cytochromes. Biochemistry 34, 15191-15203.

Daltrop, O., Allen, J.W.A., Willis, A.C., and Ferguson, S.J. (2002a). In vitro formation of a c-type cytochrome. Proc Natl Acad Sci U S A 99, 7872-7876.

Daltrop, O., Stevens, J.M., Higham, C.W., and Ferguson, S.J. (2002b). The CcmE protein of the $c$-type cytochrome biogenesis system: unusual in vitro heme incorporation into apo-CcmE and transfer from holo-CcmE to apo-cytochrome. Proc Natl Acad Sci U S A 99, 9703-9708.

Enggist, E., Thöny-Meyer, L., Güntert, P., and Pervushin, K. (2002). NMR structure of the heme chaperone CcmE reveals a novel functional motif. Structure 10, 1551-1557.

Fee, J.A., Todaro, T.R., Luna, E., Sanders, D., Hunsicker-Wang, L.M., Patel, K.M., Bren, K.L., Gomez-Moran, E., Hill, M.G., Ai, J., et al. (2004). Cytochrome $r_{552}$, formed during expression of the truncated, Thermus thermophilus cytochrome $c_{552}$ gene in the cytoplasm of Escherichia coli, reacts spontaneously to form protein-bound 2-formyl-4-vinyl (Spirographis) heme. Biochemistry 43, 12162-12176.

Feissner, R.E., Richard-Fogal, C.L., Frawley, E.R., and Kranz, R.G. (2006). ABC transporter-mediated release of a haem chaperone allows cytochrome $c$ biogenesis. Mol Microbiol 61, 219-231.

Kranz, R., Lill, R., Goldman, B., Bonnard, G., and Merchant, S. (1998). Molecular mechanisms of cytochrome $c$ biogenesis: three distinct systems. Mol Microbiol 29, 383-396.

Kranz, R.G., Richard-Fogal, C., Taylor, J.-S., and Frawley, E.R. (2009). Cytochrome $c$ biogenesis: mechanisms for covalent modifications and trafficking of heme and for heme-iron redox control. Microbiol Mol Biol Rev 73, 510-528.

Liu, X., Kim, C.N., Yang, J., Jemmerson, R., and Wang, X. (1996). Induction of apoptotic program in cell-free extracts: requirement for dATP and cytochrome $c$. Cell 86, 147-157.

Richard-Fogal, C.L., Frawley, E.R., Bonner, E.R., Zhu, H., San Fran- 
cisco, B., and Kranz, R.G. (2009). A conserved haem redox and trafficking pathway for cofactor attachment. EMBO J 28, 2349-2359.

Richard-Fogal, C.L., Frawley, E.R., and Kranz, R.G. (2008). Topology and function of CcmD in cytochrome $c$ maturation. J Bacteriol 190, 3489-3493.

San Francisco, B., Bretsnyder, E.C., Rodgers, K.R., and Kranz, R.G. (2011). Heme ligand identification and redox properties of the cytochrome $c$ synthetase, CcmF. Biochemistry 50, 10974-10985.

Sawyer, E.B., Stephens, E., Ferguson, S.J., Allen, J.W.A., and Barker, P.D. (2010). Aberrant attachment of heme to cytochrome by the $\mathrm{Ccm}$ system results in a cysteine persulfide linkage. J Am
Chem Soc 132, 4974-4975.

Schulz, H., Hennecke, H., and Thöny-Meyer, L. (1998). Prototype of a heme chaperone essential for cytochrome $c$ maturation. Science 281, 1197-1200.

Stevens, J.M., Mavridou, D.A.I., Hamer, R., Kritsiligkou, P., Goddard, A.D., and Ferguson, S.J. (2011). Cytochrome $c$ biogenesis System I. FEBS J 278, 4170-4178.

Thöny-Meyer, L. (2002). Cytochrome $c$ maturation: a complex pathway for a simple task? Biochem Soc Trans 30, 633-638.

Thöny-Meyer, L., Fischer, F., Künzler, P., Ritz, D., and Hennecke, H. (1995). Escherichia coli genes required for cytochrome $c$ maturation. J Bacteriol 177, 4321-4326. 